\title{
Comparison between direct chairside and digitally fabricated temporary crowns
}

\author{
Adil O. ABDULLAH ${ }^{1,2}$, Sarah POLLINGTON² and Yi LIU1 \\ ${ }^{1}$ Stomatology Research Center, School of Stomatology, China Medical University, Shenyang, Liaoning Province, China \\ ${ }^{2}$ Academic Unit of Restorative Dentistry, School of Clinical Dentistry, the University of Sheffield, Sheffield, UK \\ Corresponding author, Yi LIU; E-mail: liuyi@cmu.edu.cn
}

\begin{abstract}
The mechanical properties of temporary crowns are considered to be crucial in order to achieve successful definite restorations. This study compared marginal fit, internal fit, fracture strength and mode of fracture of CAD/CAM temporary crowns to direct chairside counterparts. An upper left first premolar Frasaco tooth was prepared for all-ceramic crown. The materials used for comparison were VITA CAD-Temp ${ }^{\circledR}$, ArtBloc ${ }^{\circledR}$ Temp, PMMA DISK and Acrytemp (control group). The crowns were divided into four groups ( $n=10$ ). Each crown was investigated for the above parameters. Statistical analysis was performed using SPSS v.20. The average marginal gap, internal gap and fracture strength showed statistically significant difference between groups $(p<0.01)$. The fracture mode showed statistically non-significant difference $(p>0.05)$ among experimental groups. The CAD/CAM temporary crowns demonstrated superior mechanical properties compared to direct handmade counterparts.
\end{abstract}

Keywords: CAD/CAM, Fixed temporary prosthesis, Marginal and internal gap, Fracture strength, Dental crown

\section{INTRODUCTION}

The demand for tooth colored restorations has increased significantly in recent years due to improved techniques, materials and also patient demand for aesthetic restorations. Therefore, using various modern restorative materials which have acceptable mechanical properties, are essential for both temporary and definitive restorations ${ }^{1)}$. A temporary restoration is an important part of prosthetic therapy procedures with fixed prostheses (i.e., crowns and bridges) ${ }^{2}$. It can be used as an intermediate stage for short or longterm placement on teeth between the time of tooth preparation until the definitive indirect restorations are fitted $^{3)}$. Accurate temporary restorations are essential and serve various functions, including protection of the pulpal tissues, preventing bacterial contamination and preservation of the periodontal tissues ${ }^{4-7}$. Therefore, well fabricated temporary restorations should provide a preview of the future prosthesis and enhance the health of the abutments and periodontium ${ }^{8}$.

Polymethyl methacrylate (PMMA) resins and composite-based resins (CBR) are the most common materials used to fabricate temporary restorations ${ }^{9}$. Previous studies have shown that insufficient mechanical properties are common causes of failure of temporary restorations such as fracture and poorlyfabricated prostheses ${ }^{10)}$. These issues can cause patient discomfort and pain in addition to economic loss ${ }^{11,12)}$. Thus, the mechanical properties of temporary materials are important and should be considered to ensure the clinical success of final restoration ${ }^{13)}$.

$\mathrm{CAD} / \mathrm{CAM}$ technology, which is used to fabricate temporary and definitive prosthesis, may solve some of

Color figures can be viewed in the online issue, which is available at J-STAGE.

Received Sep 4, 2017: Accepted Jan 24, 2018

doi:10.4012/dmj.2017-315 JOI JST.JSTAGE/dmj/2017-315 these issues ${ }^{14)}$. CAD/CAM-fabricated temporary crowns could provide better outcomes regarding marginal fit, strength and fabrication in a shorter time ${ }^{14)}$. In addition, the emergence of the technology in dentistry has allowed the successful application of various superior materials ${ }^{15)}$. Using CAD/CAM systems to fabricate temporary prosthesis have now achieved more popularity in comparison to conventional counterpart ${ }^{1)}$. This technology permits shaping of materials with high precision that cannot be carried out effectively through a traditional method ${ }^{16)}$.

At present, there are few studies that have been published comparing the efficacy of the marginal fit, internal fit, fracture strength and mode of fracture of direct handmade temporary prosthesis relative to those fabricated with the CAD/CAM technique ${ }^{17,18)}$. Therefore, the primary objective of the present study was to compare the marginal fit, internal fit, fracture strength and mode of fracture of handmade fabricated temporary crowns with those of digitally fabricated crowns. The null hypothesis was twofold: it was hypothesized that digitally fabricated temporary crowns would provide better adaptation than direct handmade counterparts. In addition, it was also hypothesized that there is no difference between digitally fabricated and direct handmade temporary crowns in terms of fracture resistance property.

\section{MATERIALS AND METHODS}

An upper left first premolar phantom tooth on a model (Frasaco Franz Sachs, Tettnang, Germany) was used for tooth preparation. The protocol for all-ceramic preparation was followed with $1.5 \mathrm{~mm}$ occlusal reduction, the convergence angle of the wall was prepared to be approximately $6^{\circ}$ and a round shoulder of $1 \mathrm{~mm}$ using 
a high speed handpiece operating with water coolant. A silicone index was used to permit easy visual inspection and provided stability during tooth preparation. The Williams periodontal probe was used to measure the preparation dimensions as per the protocol. A parallelometer was also used to achieve a standardised tooth preparation. An impression of the prepared tooth was made with vinyl-polysiloxane (Dublisil 15, Dreve Dentamid, Unna, Germany) to fabricate a die model from polyurethane base resin (AlphaDie ${ }^{\mathrm{TM}} \mathrm{MF}$, Schütz Dental Labortechnik, Rosbach, Germany).

The CEREC4 InEos system (Sirona, Bensheim, Germany) was used for scanning the preparation. The preparation was powdered with scan spray lab (Dentaco, Essen, Germany) to provide optimum scanning process. The preparation was then scanned and the crown designed using the CEREC4 SW4.4.1. Three CAD/CAM temporary blocks were used: VITA CAD-Temp ${ }^{\circledR}$ (VITA Zahnfabrik, H. Rauter, Bad Säckingen, Germany), ArtBloc ${ }^{\circledR}$ Temp (Merz Dental, Lütjenburg, Germany), and PMMA DISK (YAMAHACHI DENTAL MFG, Gamagori, Japan). Ten temporary crowns were milled for each group. The correlation mode was used with the spacer set at 10 microns for the three CAD/CAM groups and the default burs (1.2 mm cylinder bur and step bur) were used for the milling process. Following milling, the excess handles of the crown and the surface was smoothed with a carbide finishing bur. The restorations were examined for the presence of any defects or cracks under a stereomicroscope (MEIJI Techno, RZ Series Zoom Stereo Microscope, Saitama, Japan).

A fourth group which consisted of temporary crowns were made from Acrytemp (Zhermack, Badia Polesine, Italy), using the direct fabrication technique. An impression of the upper left first premolar phantom tooth on Frasaco model was initially taken before preparation to serve as an index for the fabrication of provisional crowns. The materials used were heavy body material (Aquasil putty, blue color, Dentsply-DeTrey, Constanz, Germany) and light body material (Aquasil LV'TM green color, Denstply-DeTrey) as per the manufacturer's instructions. To achieve an accurate record, the impression material extended beyond gingival margin and adjacent tooth on either side used a sectional tray. Once the impression was set, it was then carefully removed and checked for any defects. Any excess was removed with a blade No.25 and scalpel.

Next petroleum jelly was applied to the proximal areas of adjacent teeth to allow easy removal of the crown from the seated position. The components of the Acrytemp restorative material were mixed in an automixing gun with $4: 1$ base/catalyst ratio and injected gently around the margins of the prepared tooth, followed injecting into the indexed impression. The impression silicone was carefully loaded and seated onto the prepared tooth with finger pressure occlusally to ensure adequate flow of temporary material on the prepared tooth. After $4 \mathrm{~min}$ from starting application of the Acrytemp at normal room temperature, the indexed impression was then removed. After that period, the direct temporary crown reached its elastic stage and permitted removal from the prepared tooth used plastic instrument with a gentle pressure at proximal sides.

To allow complete polymerization and hardening of the Acrytemp, the crowns were left at room temperature for $10 \mathrm{~min}$. They were then checked for accurate adaptation followed by rubbing with ethyl alcohol to remove the oxygen inhibition layer. In addition, a digital caliper (Pearson Dental ${ }^{\mathrm{TM}}$, Vivid Labpro Dial Caliper, K2103, Y0-01201, Sylmar, CA, USA) was used to check the thickness and uniformity of manually prepared temporary crowns. All crowns were checked at different points such as buccal, palatal, mesial, distal, occlusal, axial, and marginal areas for accuracy. The ten direct temporary crowns were made by one operator. The crowns were then finished and polished used rotary rubber cups (Astropol, Ivoclar Vivadent, Schaan, Liechtenstein) and inspected for any deformities circumferentially.

\section{Measurement of marginal and internal fit}

The marginal gap was evaluated by means of a replica technique and a luting agent. A light-bodied silicone rubber impression material (Aquasil $\mathrm{LV}^{\mathrm{TM}}$, green color, Dentsply-DeTrey) was used for cementation. Each crown was filled with the light-bodied material and placed on the corresponding replica with a constant force of $40 \mathrm{~N}$ for 3 min using a universal testing machine (EZ test, Shimadzu, Kyoto, Japan). After setting of the silicone rubber, the crowns were removed from the die model. A thin film of the light bodied impression material was adhered to the inner surface of the crown in all cases. To support the thin film, a heavy bodied materials of contrasting colors (Aquasil putty, blue and orange color, Dentsply-DeTrey) was used. After setting, an index was marked on the heavy-bodied material to provide a consistent series of locations (mesial, distal, buccal, and palatal) for sectioning, and each silicone replica was sectioned into smaller segments for microscopic examination. The marginal gap and internal fit were measured at nine points seperately both buccopalatally [BP] and mesiodistally [MD]. In each specimen, 18 measurements were evaluated, amounting to a total of 720 measurements for four groups $(n=40)$. The schematic illustration of silicone replica technique, which measured different points at interface cross sectional view from $[\mathrm{BP}]$ and $[\mathrm{MD}]$ directions, is shown in Fig. 1 using the stereomicroscope with $25 \times$ magnification.

\section{Measurement of fracture strength}

To perform the test a die model was fabricated to subject the fracture strength testing. To duplicate the prepared tooth and fabricate a die model, a convenient wax base was fabricated for the prepared tooth. The root portion of the Frasaco tooth was embedded into the wax. The full crown area was left on the outside of the wax base. The height and width of the base was approximately 14 and $20 \mathrm{~mm}$ respectively. A plastic cylinder was used to fabricate a new impression of the prepared tooth. The height and diameter of the cylinder was 50 and $30 \mathrm{~mm}$ respectively. Then the embedded prepared tooth was 


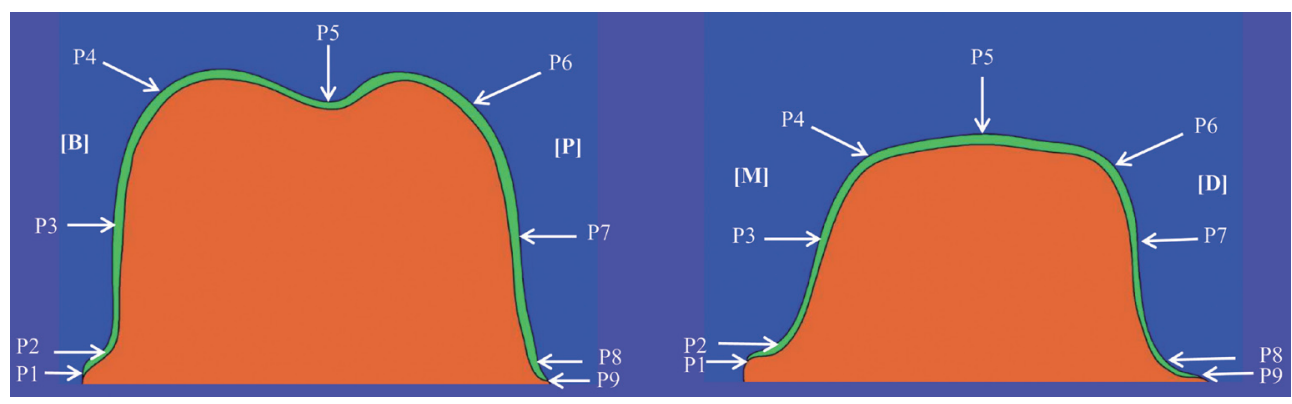

Fig. 1 Schematic illustration of the segmented cross sectional view of the silicone replica technique.

The blue and orange colors represent heavy body materials and the thin green layer in between represents light body material by means of cement film thickness. The cross sectional views from buccopalatal [BP] and mesiodistal [MD] directions were measured in different points $(\mathrm{P})$ : (P1, P9): marginal seat; (P2, P8): marginal angle; (P3; P7): axial wall; (P4, P6): cusp tip; (P5): occlusal plateau.

Table 1 Illustrates the pattern of crown fracture

\begin{tabular}{cl}
\hline Types of fracture & \multicolumn{1}{c}{ Description of fracture pattern } \\
\hline Type I & Minimal fracture or crack in crown \\
Type II & Less than half of crown lost \\
Type III & Crown fracture through midline; half of crown displaced or lost \\
Type IV & More than half of crown lost \\
\hline
\end{tabular}

placed and fixed at the bottom of the cylinder.

Next, the addition of vinyl-polysiloxane (Dublisil 15, Dreve Dentamid) impression material was used to fabricate a model impression mold for the prepared Frasaco tooth. The impression material consists of $(\mathrm{A}+\mathrm{B})$ components, the digital scaler (VIBRA AJ, SHINKO DENSHI, Tokyo, Japan) was used to weight an adequate amount of components into the equal ratio following the manufacturer's instructions. Mixing of the components was carried out in a plastic dish with a small plastic spatula for $45 \mathrm{~s}$ at normal room temperature. For each measurement the equipment was re-zeroed to achieve an optimum accuracy during measurement.

The mixed pastes were poured into the plastic cylinder and left for $30 \mathrm{~min}$ to set. The impression was then removed from the cylinder after dissection of the plastic cylinder from a side using a scalpel and No. 25 blade to release the impression free from distortion and tearing. Then, the fabricated silicone mold was separated from the tooth and its wax base.

After fabrication of a silicone mold, a die model was duplicated using polyurethane base resin (AlphaDie ${ }^{\mathrm{TM}}$ MF, Schütz Labortechnik). The material consisted of three main components: $200 \mathrm{~mL}$ base material, 100 $\mathrm{mL}$ hardener and $400 \mathrm{~g}$ filler. The contents were then weighed using a digital scaler and mixed. The mixing constituents' ratio were prepared as a base: hardener (2:1) with approximately $15 \mathrm{~g}$ AlphaDie ${ }^{\mathrm{TM}} \mathrm{MF}$. The mixing process was carried out in a plastic dish used a plastic spatula for about $30 \mathrm{~s}$ at normal room temperature. The mixture was poured into the prepared mold and left for approximately $1 \mathrm{~h}$ to permit the setting process. The die model was then removed, and the excess borders were trimmed to adequate size.

Then, each crown was cemented to the die model using Integrity ${ }^{\circledR}$ TempGrip ${ }^{\circledR}$ (Dentsply Caulk, Milford, DE, USA). The components were mixed in dual barreled syringe with a material ratio of $1: 1$, following the manufacturer's instruction to achieve a standardised time to allow complete setting of the cement. The crowns were filled with cement and seated on its corresponding die model which was represented as a prepared Frasaco tooth. The cemented crowns were immersed into water for $24 \mathrm{~h}$ to simulated an oral environment and subjected to static loading.

The samples were loaded under a standard compression load at a crosshead speed of $1 \mathrm{~mm} / \mathrm{min}$ and the force recorded using the universal testing machine (EZ test, Shimadzu) with a 2,000 N loaded cell for 3 min. A plunger with a steel ball (ø $4.24 \mathrm{~mm}$ ) was used to apply a compressive force until fracture occurred. The ball was directed to the mid occlusal plateau between the buccal and palatal cusps. A piece of rubber dam was placed as a stress breaker between occlusal surface of each crown and steel ball to reduce any potential stress concentration while applying the load. Loading was continued until fracture occurred and fracture loads were recorded. After a first loading test, the die model 
was examined microscopically using stereomicroscope to detect any deformation or cracks from the occlusal surface and finishing line circumferentially.

The mode of fracture detected was also recorded for the samples. The record based on Burke's classification, which comprises certain fracture types as shown in Table 1.

\section{Statistical analysis}

All data about the different fabrication techniques and materials used were analyzed using IBM SPSS v.20 (IBM Statistical Package for Social Sciences, Armonk, NY, USA). The mean and standard deviations of the marginal gap, internal fit, and fracture strength were calculated used one-way analysis of variance (ANOVA) followed by post hoc tests (Tukey's test). The data of mode of fracture were compared using Chi-square test. The $p$-value less than $0.05(p<0.05)$ was considered as statistically significant.

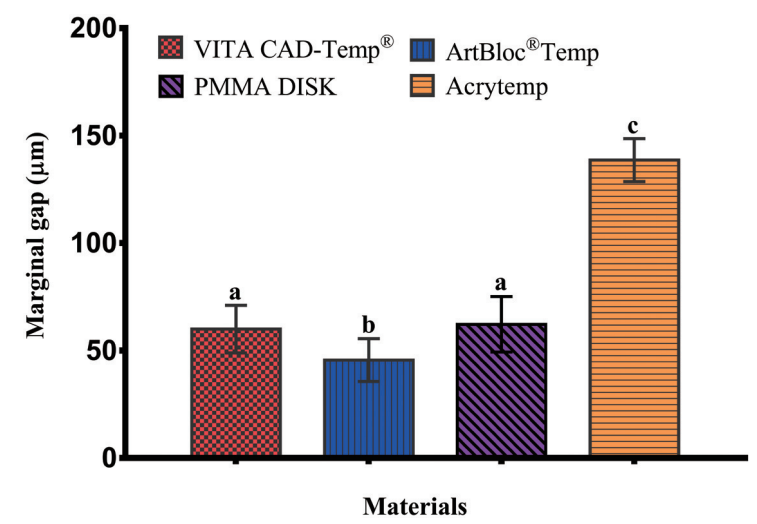

Fig. 2 Bar graph showing the mean values and SD of the marginal gap in $(\mu \mathrm{m})$ for each group of material. Means with same letters are not significantly different. Means with different letters are significantly different.

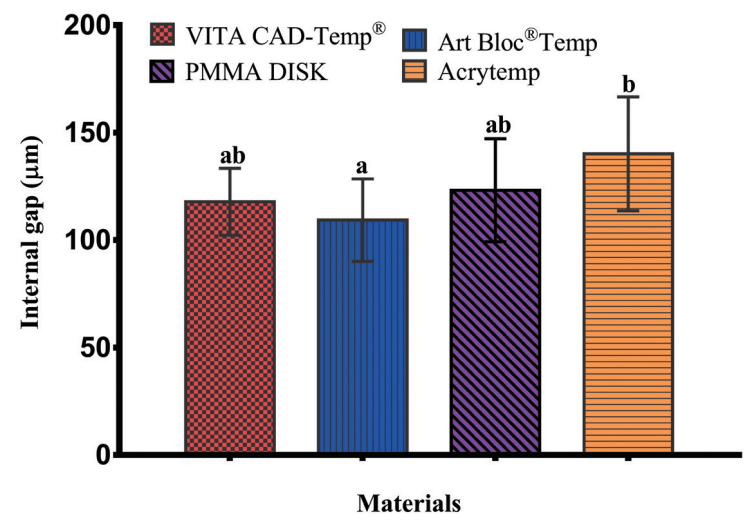

Fig. 3 Bar graph showing the mean values and SD of the internal gap in $(\mu \mathrm{m})$ for each group of material. Means with same letters are not significantly different. Means with different letters are significantly different.

\section{RESULTS}

Mean values and standard deviations $(\mathrm{M} \pm \mathrm{SD})$ of the marginal gap, internal gap and fracture strength for all groups are shown graphically in Figs. 2-5.

The average marginal gap for each group was: VITA CAD-Temp $^{\circledR} 59.97 \pm 11.1 \mu \mathrm{m}$, ArtBloc ${ }^{\circledR}$ Temp $45.58 \pm 9.99$ $\mu \mathrm{m}, \quad$ PMMA DISK 62.19 $\pm 12.9 \mu \mathrm{m}$ and Acrytemp $138.6 \pm 10.1 \mu \mathrm{m}$. The statistical analysis of the results indicated that there was a statistically significant difference $(p<0.001)$ between the groups. The average internal fit for each group was: VITA CAD-Temp ${ }^{\circledR}$ group $117.8 \pm 15.58 \mu \mathrm{m}$, ArtBloc ${ }^{\circledR}$ Temp group $109.27 \pm 19.21 \mu \mathrm{m}$, PMMA DISK group 123.16 $\pm 23.97 \mu \mathrm{m}$ and Acrytemp group $140.1 \pm 26.53 \mu \mathrm{m}$. The results of the internal fit between all groups showed a statistically significant difference $(p<0.001)$. The average fracture strength of each group was: VITA CAD-Temp ${ }^{\circledR} 347 \pm 30.71$ Newton

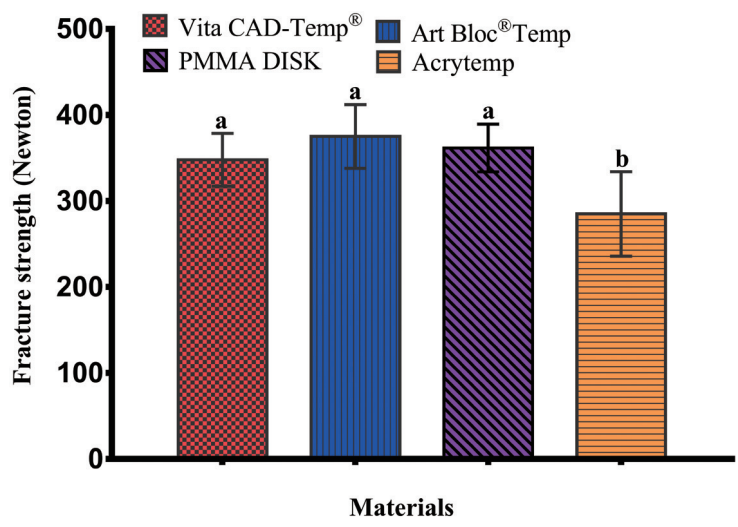

Fig. 4 Bar graph showing the mean values and SD of the fracture strength in (Newton) for each group of material.

Means with same letters are not significantly different. Means with different letters are significantly different.

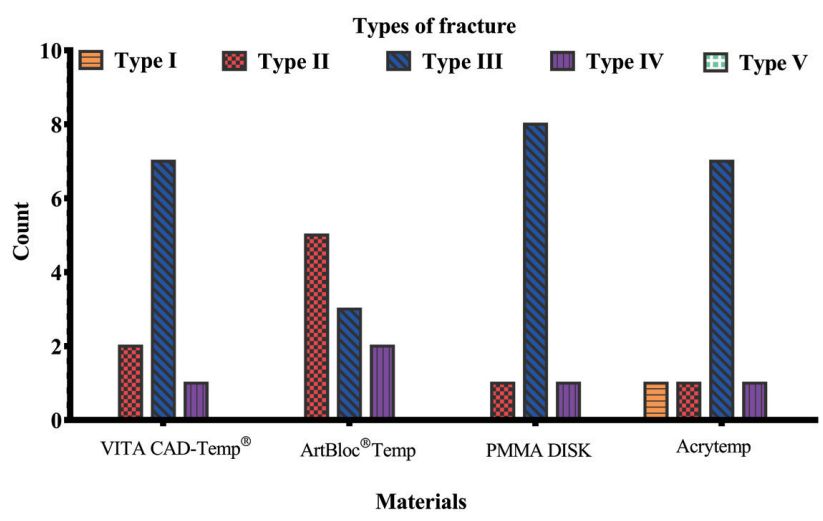

Fig. 5 Bar graph showing the mode of failure for each material.

For all groups, the majority of the crowns had type II and type III fractures. 
(N), ArtBloc ${ }^{\circledR}$ Temp $375.04 \pm 36.97$ N, PMMA DISK

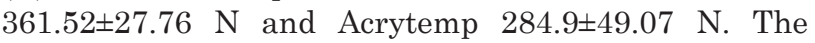
results of the fracture strength between all groups showed a statistically significant difference $(p<0.001)$.

One-way ANOVA indicated that for the variables; marginal gap, internal gap and fracture resistance, the $(p<0.05)$ was statistically significant. The result of fracture mode showed that there was no statistically significant difference between investigated groups, $p$-value greater than 0.05 as shown in Fig. 5 .

\section{DISCUSSION}

The aim of this study was to compare the digitally fabricated temporary crowns to direct handmade counterparts. Previous studies have reported that the digitally fabricated temporary restorations might be superior to the direct conventional method ${ }^{17,18}$. The advancement in CAD/CAM technology permitted milling of the blocks with satisfied clinical performance and an easier approach for crown fabrication ${ }^{19-21)}$. This technology is not without shortcomings regarding fabrication of temporary restorations such as uneven preparation of crowns internally, particularly at the axial walls and occlusal plateau ${ }^{17,22}$. Other studies have been reported that this technology and its materials are more reliable as an alternative to conventional handmade technique $^{23)}$. In addition, CAD/CAM technology was progressed rapidly in the last three decades, with the initial limits of the chairside system having been overcome $\mathrm{e}^{24)}$.

Certain parameters can govern the success and failure rates for fabricating temporary and permanent restorations such as marginal and internal accuracies ${ }^{25}$. When a satisfactory marginal and internal fit has been achieved following fabrication crowns, it can be considered as a successful process ${ }^{17}$, particularly, if the material can withstand the masticatory forces in oral environment.

Regarding the marginal adaptation, this is a crucial aspect, which should be considered carefully. Poor marginal adaptation will lead to damage of the surrounding tooth tissues, a situation that deteriorates the complete restoration seriously, causing exposed margins and poor aesthetics ${ }^{26)}$. Therefore, it is essential to fabricate temporary crowns in a way that will harmonize with the surrounding tissues until final restoration is placed.

The current study used a replica method to measure the gaps that were formed through digital and handmade fabrication temporary crowns. For measuring marginal and internal accuracies, there are various methods, which can be replica, embedded or sectioning techniques ${ }^{27}$. The replica technique is a non-invasive and valid approach to measure the adaptation of a restoration to the tooth structure $^{28}$.

The current research has recorded the mean and standard deviations to evaluate fit accuracies of specimens from diverse points marginally and internally. The average marginal gap of digitally fabricated temporary crowns reported in the current study ranged from 46-138 $\mu \mathrm{m}$. The previous study has recorded marginal gaps ranged between $55-193 \mu \mathrm{m}$ as different materials and digital system were used ${ }^{17}$. Despite achieving better results, however, the film thickness is still far from the basic requirements which was reported the range from $25-40 \mu \mathrm{m}^{29)}$.

There was statistically significant difference between digitally fabricated crowns to the handmade counterpart $(p<0.001)$ when measuring marginal fit, with the former showing better marginal adaptation than the latter. The highest marginal opening was with Acrytemp $138 \mu \mathrm{m}$ (control group). It is also interesting to note that PMMA DISK crowns have shown the lowest marginal integrity, while the ArtBloc $^{\circledR}$ has demonstrated the highest marginal fit among CAD/CAM temporary blocks. These outcomes have not been described previously.

Previous studies have been reported acceptable range of marginal opening for clinical cases which is less than $120 \mu \mathrm{m}^{28,29)}$. The CAD/CAM materials in the present study could show the acceptable range of marginal gaps. However, handmade crowns have exceeded the mentioned range. The current result is consistent with previously reported literature ${ }^{177}$.

The current outcomes of the digitally fabricated temporary crowns relating to marginal accuracy is consistent to permanent fabricated crowns such as zirconia and lithium disilicate materials that could provide marginal fit which considered within the clinical acceptable range ${ }^{30,31)}$. However, this is not the case for temporary handmade crowns as they have shown larger marginal openings. Therefore, it is possible to keep tooth tissues and its surrounding structures with digitally fabricated crowns until the temporary restoration will be replaced with a definite crown. Thus, the handmade temporary crowns should be used with caution especially when used as a long term temporary crown.

There is another shortcoming regarding polymerization shrinkage among temporary handmade crowns ${ }^{12)}$. This might be a causative factor for marginal discrepancy among temporary handmade crowns. This potential issue cannot be observed in CAD/CAM blocks. During the milling process, the blocks are not affected as they are completely pre-polymerized during fabrication process prior to machining ${ }^{32,33}$. The present study has recorded better results than earlier investigations regarding shrinkage issue ${ }^{34}$.

The use of silicone impression material, provides certain promising properties such as dimensional stability and accurate recording of contours ${ }^{35}$. However, temporary handmade crowns presented with greater marginal openings than the digitally fabricated crowns. This fact may contribute to the shrinkage potential of handmade temporary crowns that was mentioned earlier.

Regarding the internal fit, the present data have shown $109-140 \mu \mathrm{m}$ as an average internal gap. There was a statistically significant difference between evaluated groups $(p<0.001)$. The ArtBloc ${ }^{\mathbb{E}}$ Temp temporary blocks demonstrated better internal fit. Contrary, the highest 
internal gap was recorded with Acrytemp handmade crowns. The present study has recorded lower internal gaps among CAD/CAM and handmade temporary crowns compared to previous literature ${ }^{17}$.

The temporary cement was used to fix the crowns on the die model to simulate the oral environment. The outcome of the fracture strength has shown statistically significant differences between all tested groups $(p<0.001)$. The mean fracture strength reported in the current study ranged from 284-375 N. The ArtBloc ${ }^{\circledR}$ Temp demonstrated the highest fracture resistance among CAD/CAM temporaries. In the current investigation, VITA CAD-Temp has shown the lowest fracture resistance among CAD/CAM blocks $347 \mathrm{~N}$. However, the amount of resistance force is still greater than the amount that applied to the direct handmade counterpart $284 \mathrm{~N}$.

All tested materials were subjected to a load that was higher than the masticatory forces inside the individuals mouth. It has been reported that the force produced in the mouth varies between individuals. For example, different forces such as $40 \mathrm{~N} ; 170-881 \mathrm{~N}$; and 200-540 $\mathrm{N}$ have been recorded during swallowing; chewing nuts; and forces increase in the molar region, respectively ${ }^{36}$.

Regarding the fracture mode, the analyzed data have shown that there was no statistically significant difference between digital and direct handmade temporary crowns $(p>0.05)$. The highest number of the fractured pattern was recorded as type II fracture (less than half of crown lost) and type III fracture (crown fracture through midline; half of crown displace or lost). This finding is inconsistent with earlier literature as the study reported type II fracture as a common pattern of crown fracture ${ }^{17)}$.

It is important to note that the average cyclic load during an individual lifespan in actual oral environment may exceed $1 \times 10^{7}$ cyclic load $^{37}$, which may lead to $50 \%$ or more strength reduction ${ }^{38)}$ Therefore, one of the limitations of the current study was that it was not used fatigue loading for the crowns before measuring fracture strength.

The clinical significance of this investigation is that the current outcomes could draw clinicians' attention to the importance of selecting the right material for clinical cases. In addition, it provides a clear concept for clinicians to opt more accurate materials for fabricating temporary crowns.

\section{CONCLUSION}

Within the limitations of this study, the first part of the null hypothesis was accepted. The digitally fabricated temporary crowns demonstrated superior fit in comparison to direct handmade temporary crowns. The second part of the null hypothesis was rejected as there was a statistically significant difference between digitally fabricated and direct handmade temporary crowns in terms of fracture strength; the former materials have shown superior resistance.

\section{ACKNOWLEDGMENTS}

The authors would like to express their heartfelt gratitude to Chinese Government Scholarship Council (CSC) for providing the grant to conduct the research. The Number of grant is (CSC-2016368012).

\section{CONFLICTS OF INTEREST}

The authors report no conflicts of interest.

\section{REFERENCES}

1) Alt V, Hannig M, Wostmann B, Balkenhol M. Fracture strength of temporary fixed partial dentures: CAD/CAM versus directly fabricated restorations. Dent Mater 2011; 27: 339-347.

2) Herbert T. Shillingburg DAS, Edwin L. Wilson, Joseph R. Cain, Donald L. Mitchell, Luis J. Blanco, James C. Kessler. Fundamentals of fixed prosthodontics. 4 ed: Auflage. Chicago: Quintessence; 2012.

3) Beyari MM. Marginal and internal crown fit evaluation of $\mathrm{CAD} / \mathrm{CAM}$ versus press-laboratory all-ceramic crown. Clin Med Diag 2014; 4: 21-26.

4) Nugala B, Kumar BBS, Sahitya S, Krishna PM. Biologic width and its importance in periodontal and restorative dentistry. J Conserv Dent 2012; 15: 12-17.

5) Singh S, Tripathi J, Rai N. An appraisal of the bioavailability enhancers in Ayurveda in the light of recent pharmacological advances. J Interdiscip Dent 2012; 2: 3-10.

6) Shaveta Sood SG. Periodontal-restorative interactions: A review. Indian J Clin Pract 2013; 23: 707-713.

7) Ortega R, Gonzalo E, Gomez-Polo M, Lopez-Suarez C, Suarez MJ. SEM evaluation of the precision of fit of CAD/CAM zirconia and metal-ceramic posterior crowns. Dent Mater J 2017; 36: 387-393.

8) Saisadan D, Manimaran P, Meenapriya PK. In vitro comparative evaluation of mechanical properties of temporary restorative materials used in fixed partial denture. J Pharm Bioall Sci 2016; 8: S105-109.

9) Burns DR, Beck DA, Nelson SK. A review of selected dental literature on contemporary provisional fixed prosthodontic treatment: report of the Committee on Research in Fixed Prosthodontics of the Academy of Fixed Prosthodontics. J Prosthet Dent 2003; 90: 474-497.

10) Kim SH, Watts DC. In vitro study of edge-strength of provisional polymer-based crown and fixed partial denture materials. Dent Mater 2007; 23: 1570-1573.

11) Balkenhol M, Ferger P, Mautner MC, Wostmann B. Provisional crown and fixed partial denture materials: mechanical properties and degree of conversion. Dent Mater 2007; 23: 1574-1583.

12) Balkenhol M, Knapp M, Ferger P, Heun U, Wostmann B. Correlation between polymerization shrinkage and marginal fit of temporary crowns. Dent Mater 2008; 24: 1575-1584.

13) Tsitrou E, Helvatjoglou-Antoniades M, Pahinis K, van Noort R. Fracture strength of minimally prepared resin bonded CEREC inlays. Oper Dent 2009; 34: 537-543.

14) Karaokutan I, Sayin G, Kara O. In vitro study of fracture strength of provisional crown materials. J Adv Prosthodont 2015; 7: 27-31.

15) Fasbinder DJ. Chairside CAD/CAM: an overview of restorative material options. Compend Contin Educ Dent 2012; 33: 50, $52-58$.

16) Sakornwimon N, Leevailoj C. Clinical marginal fit of zirconia crowns and patients' preferences for impression techniques using intraoral digital scanner versus polyvinyl siloxane 
material. J Prosthet Dent 2017; 118: 386-391.

17) Abdullah $\mathrm{AO}$, Tsitrou EA, Pollington S. Comparative in vitro evaluation of $\mathrm{CAD} / \mathrm{CAM}$ vs conventional provisional crowns. J Appl Oral Sci 2016; 24: 258-263.

18) Rayyan MM, Aboushelib M, Sayed NM, Ibrahim A, Jimbo R. Comparison of interim restorations fabricated by CAD/CAM with those fabricated manually. J Prosthet Dent 2015; 114: 414-419.

19) Schweyen R, Beuer F, Bochskanl M, Hey J. Implementing a new curriculum for computer-assisted restorations in prosthetic dentistry. Eur J Dent Educ 2018; 22: e237-e247.

20) Li R, Jiang T, Wang YN, Li SQ, Cheng XR. Clinical evaluation and comparison of porcelain laminate veneers and computer aided design and computer aided manufacture veneers. Zhonghua kou qiang yi xue za zhi 2007; 42: 330-332.

21) Nejatidanesh F, Amjadi M, Akouchekian M, Savabi O. Clinical performance of CEREC AC Bluecam conservative ceramic restorations after five years - A retrospective study. J Dent 2015; 43: 1076-1082.

22) Coelho PG, Silva NR, Bonfante EA, Guess PC, Rekow ED, Thompson VP. Fatigue testing of two porcelain-zirconia allceramic crown systems. Dent Mater 2009; 25: 1122-1127.

23) Sannino G, Germano F, Arcuri L, Bigelli E, Arcuri C, Barlattani A. CEREC CAD/CAM chairside system. Oral Implant 2014; 7: 57-70.

24) Mormann WH. The evolution of the CEREC system. J Am Dent Assoc 2006; 137: Suppl 7s-13s.

25) Park SH, Yoo YJ, Shin YJ, Cho BH, Baek SH. Marginal and internal fit of nano-composite CAD/CAM restorations. Restor Dent Endod 2016; 41: 37-43.

26) Mitchell CA, Pintado MR, Douglas WH. Nondestructive, in vitro quantification of crown margins. J Prosthet Dent 2001; 85: 575-584.

27) Han HS, Yang HS, Lim HP, Park YJ. Marginal accuracy and internal fit of machine-milled and cast titanium crowns. J Prosthet Dent 2011; 106: 191-197.

28) Ha SJ, Cho JH. Comparison of the fit accuracy of zirconia- based prostheses generated by two CAD/CAM systems. J Adv Prosthodont 2016; 8: 439-448.

29) McLean JW, von Fraunhofer JA. The estimation of cement film thickness by an in vivo technique. Brit Dent J 1971; 131: 107-111.

30) Yildiz C, VanlioĞLu BA, Evren B, Uludamar A, ÖZkan YK. Marginal-internal adaptation and fracture resistance of $\mathrm{CAD} /$ CAM crown restorations. Dent Mater J 2013; 32: 42-47.

31) Yildirim G, Uzun IH, Keles A. Evaluation of marginal and internal adaptation of hybrid and nanoceramic systems with microcomputed tomography: An in vitro study. J Prosthet Dent 2017; 118: 200-207.

32) Nejatidanesh F, Lotfi HR, Savabi O. Marginal accuracy of interim restorations fabricated from four interim autopolymerizing resins. J Prosthet Dent 2006; 95: 364-367.

33) Young HM, Smith CT, Morton D. Comparative in vitro evaluation of two provisional restorative materials. J Prosthet Dent 2001; 85: 129-132.

34) Yao J, Li J, Wang Y, Huang H. Comparison of the flexural strength and marginal accuracy of traditional and CAD/CAM interim materials before and after thermal cycling. J Prosthet Dent 2014; 112: 649-657.

35) Hamalian TA, Nasr E, Chidiac JJ. Impression materials in fixed prosthodontics: influence of choice on clinical procedure. J Prosthodont 2011; 20: 153-160.

36) De Las Casas EB, de Almeida AF, Cimini Junior CA, Gomes Pde T, Cornacchia TP, Saffar JM. Determination of tangential and normal components of oral forces. J Appl Oral Sci 2007; 15: 70-76.

37) Peterson IM, Wuttiphan S, Lawn BR, Chyung K. Role of microstructure on contact damage and strength degradation of micaceous glass-ceramics. Dent Mater 1998; 14: 80-89.

38) Penate L, Basilio J, Roig M, Mercade M. Comparative study of interim materials for direct fixed dental prostheses and their fabrication with CAD/CAM technique. J Prosthet Dent 2015; 114: 248-253. 